\title{
Delayed diagnosis of disorder of sex development (DSD) due to P450 oxidoreductase (POR) deficiency
}

\author{
Vasiliki Koika, Anastasia K. Armeni, Neoklis A. Georgopoulos \\ Division of Reproductive Endocrinology, Department of Obstetrics and Gynecology, University of Patras Medical School, \\ Patras, Greece
}

\begin{abstract}
CASE PRESENTATION: A 36-year old man, operated on for cryptorchidism at the age of 8 years, was referred to the Outpatient Clinic of Reproductive Endocrinology for investigation of infertility. Clinical examination revealed ambiguous genitalia: penis $4-5 \mathrm{~cm}$, testicular volume 2-3 ml, hypospadias, hypertrophic foreskin and scrotum bifida. Mild hypertension was confirmed. No skeletal malformations were detected. DESIGN: Hormonal and electrolytic determinations as well as semen analysis were conducted. PCR of the coding regions of 17-hydroxylase/17,20 lyase (P450c17) and of $P 450$ oxidoreductase (POR) genes was also performed. RESULTS: Normal levels of electrolytes, low levels of androgens, high levels of gonadotropins and 17-hydroxyprogesterone as well as azoospermia were detected. Karyotype was shown to be 46,XY. Both hCG and ACTH stimulation significantly increased 17-hydroxyprogesterone with no increase in androgens. The diagnosis was congenital adrenal hyperplasia with apparent combined P450c17 and P450c21 deficiency due to mutations in the POR gene. Sequencing of the POR gene revealed: one deletion in exon 12 (Del 1696_1698delGTC >del531Valine) and one missense mutation in exon 7 (A259G) as well as two polymorphisms: rs1057868 (C/T $\mathrm{A503V}$ ) and rs1057870 (G/A S572S) in exons 12 and 13, respectively. No nucleotide changes were detected in the 8 exons of P450c17. CONCLUSIONS: Molecular findings were consistent with the diagnosis of $\mathbf{P 4 5 0}$ oxidoreductase deficiency. Despite this severe deficiency, skeletal malformations simulating Antley-Bixler syndrome, which usually characterize the most severe forms, were not confirmed. This discrepancy could be attributed to the differential impact of a POR variant on each one of the P450 enzymes.
\end{abstract}

Key words: POR deficiency, Mutation, Male pseudohermaphroditism, Disorder of sex development, DSD, Ambiguous genitalia, P450 oxidoreductase

Address for correspondence:

Neoklis A. Georgopoulos, Department of Obstetrics and Gynecology, Division of Reproductive Endocrinology,

University of Patras Medical School, University Hospital, 26500

Patras, Greece, Email: neoklisg@hol.gr, Tel:+302610999835

Received: 29-09-2015, Accepted: 09-05-2016

\section{INTRODUCTION}

Cytochrome P450 oxidoreductase deficiency (PORD) (OMIM: 613571 and OMIM: 201750) is a form of congenital adrenal hyperplasia. ${ }^{1-4}$ It was first described in 1985 in a patient with genital ambiguity 
and an abnormal urinary steroid profile, suggesting a disorder in the electron donor for $17 \alpha$-hydroxylase, 17,20 lyase and 21-hydroxylase, and was named P450 oxidoreductase. ${ }^{5}$ Although disruption of the POR gene in mice causes gross disorders of embryogenesis and embryonic lethality, ${ }^{6,7}$ in 2004 Fluck et al reported POR mutations in three children with ambiguous genitalia and skeletal malformations [Antley-Bixler syndrome (ABS)] and in a phenotypically normal adult woman with primary amenorrhea and polycystic ovaries, leading to the characterization of PORD. ${ }^{1}$ Skeletal malformation in many ABS patients with ambiguous genitalia and defective steroid metabolism have been linked to PORD, ${ }^{8}$ which is today listed as a separate metabolic disorder characterized by disruption of steroid biosynthesis in the adrenal gland and gonads. ${ }^{8-11}$

To date, more than 50 mutations have been reported, ${ }^{12,13}$ indicating that this disorder might not be uncommon. ${ }^{4,14,15}$

\section{CASE PRESENTATION}

A 36-year old-man, who was operated on for cryptorchidism at the age of 8 years, was referred to our Outpatient Clinic of Reproductive Endocrinology in 2004 for investigation of infertility. There was a negative family history of Congenital Adrenal Hyperplasia (CAH) and other cases of ambiguous genitalia/infertility within the family. The patient was not receiving any medication. He was euthyroid, with no sign of gynecomastia or BMI $27.4 \mathrm{~kg} / \mathrm{m}^{2}$ (height: $180 \mathrm{~cm}$ weight: $89 \mathrm{Kg}$ ). Clinical examination revealed a penis of 4-5 cm, testicular volume 2-3 $\mathrm{ml}$, as well as hypospadias, hypertrophic foreskin and scrotum bifida (Figure 1). Scrotal ultrasonography confirmed a testicular volume of $2 \mathrm{ml}$. Two spermiograms were performed (volume $1.5 \mathrm{ml}$ and $\mathrm{pH} 7.2$ ), revealing azoospermia.

Mild hypertension was also found. Hormonal and electrolytic determinations as well as semen analysis and karyotyping were the preliminary examinations conducted. No skeletal malformations were noted, while X-rays of the upper and lower extremities were normal. Based on the study by Krone et al, ${ }^{16}$ the malformation scoring system for PORD patients,

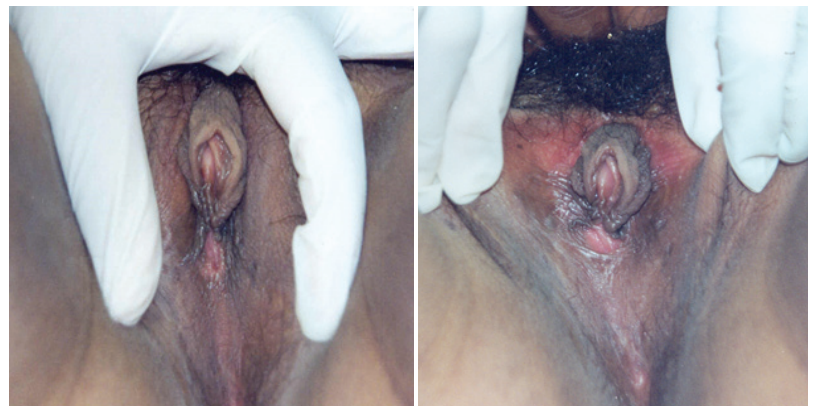

Figure 1. Pictures of the patient's external genitalia.

including midface hypoplasia, craniosynostosis, hand and feet malformations, large joint synostosis, femoral bowing, and the presence of nonskeletal malformations, was 0 . Bone densitometry with DXA revealed mild spinal cord osteopenia (z score: -1.5).

Although the patient was concerned about his fertility, after a thorough discussion he was put on testosterone treatment (IM testosterone undecanoate) in order to achieve a satisfactory sexual life. Later on, an attempt to initiate spermatogenesis was performed by hCG and FSH administration. After 18 months of treatment, testicular biopsy on both testes revealed no foci of spermatogenesis and the patient was returned to testosterone treatment.

\section{GENOTYPING}

Informed consent was obtained from the patient. Genomic DNA was extracted from peripheral blood leukocytes, followed by PCR of the coding regions of 17-hydroxylase/17,20 lyase (P450c17) and of P450 oxidoreductase (POR) genes. PCR products were subjected to sequence analysis.

The primers and the PCR reactions for the 17-hydroxylase $/ 17,20$ lyase $^{17}$ and the $\mathrm{P} 450$ oxidoreductase ${ }^{18}$ genes have been previously described. The PCR fragments were subjected to DNA sequence analysis (Macrogen Europe, Amsterdam, The Netherlands).

In order to identify the potential impact of the identified mutations on protein function, we used three free access applications: MutationTaster (http://www. mutationtaster.org/), ${ }^{19}$ SIFT (http://sift.bii.a-star.edu. sg) ${ }^{20}$ and Hansa (http://hansa.cdfd.org.in:8080/index. php?target=search). ${ }^{21,22}$ 


\section{RESULTS}

Karyotyping showed a normal male 46,XY, while semen analysis indicated azoospermia. Mild hypertension was confirmed. Biochemical tests were normal (K 4.6 and $\mathrm{Na} 144$ ), whereas the hormonal profile revealed: low levels of androgens (Testo $0.4 \mathrm{ng} / \mathrm{ml}$ (2.8-8,8), F-Testo $1.6 \mathrm{ng} / \mathrm{dl}$ (4-41), DHEAS $41 \mu \mathrm{g} / \mathrm{dl}$ $(120-360 \mu \mathrm{g} / \mathrm{dl})$, ANDRO $0.3 \mathrm{ng} / \mathrm{ml}(0.8-3.8 \mathrm{ng} / \mathrm{ml})$, high levels of Gonadotropins (LH $26 \mathrm{mIU} / \mathrm{ml}$ (1.8$12.0 \mathrm{mIU} / \mathrm{L})$, FSH $54 \mathrm{mIU} / \mathrm{ml}(1.3-19.3 \mathrm{mIU} / \mathrm{mL})$ as well as high 17-OH-progesterone levels (17-OH-Pr $11,7 \mathrm{ng} / \mathrm{ml}$ range:(0.4-3.3 ng/ml), Serum E2 concentration was $23.9 \mathrm{pg} / \mathrm{ml}$ (20-60 pg/ml). After ACTH stimulation, mild adrenal insufficiency was observed. Both hCG and ACTH stimulation substantially increased 17-hydroxyprogesterone levels (12.05ng/ $\mathrm{ml}$ and $35 \mathrm{ng} / \mathrm{ml}$, respectively) with no increase in androgens (testosterone $0.3 \mathrm{ng} / \mathrm{ml}$, DHEAS $0.6 \mu \mathrm{g} / \mathrm{dl}$ and androstenedione $0.7 \mathrm{ng} / \mathrm{ml}$ ). Baseline cortisol was $17 \mu \mathrm{g} / \mathrm{dl}$, and after stimulation $40.0(6.2-19.4 \mu \mathrm{g} / \mathrm{dl})$. Consequently, the diagnosis was congenital adrenal hyperplasia with apparent combined $\mathrm{P} 450 \mathrm{c} 17$ and P450c21 deficiency due to mutations in the POR gene.

Sequencing of POR 15 exons revealed: one deletion in exon 12 (chr7:75615089_75615091delGTC, c.1591_1593delGTC, cDNA.1696_1698delGTC, g.86572_86574delGTC del531Valine) and one novel missense mutation in exon 7 (G858C, A259G) as well as two known polymorphisms: rs1057868 (C/T A503V) and rs 1057870 (G/A S572S) in exons 12 and 13, respectively (Figure 2).

Mutation Taster identified the del531V as "disease causing”, with a probability of 0.999999999963763 , thus predicting that the amino acid sequence, the protein features as well as the splice sites would be affected. It should be mentioned that $531 \mathrm{~V}$ is a highly conserved amino acid among species and the only acceptable alternative could be Isoleucine. The A259G variant was also categorized as "disease causing", with a probability of 1 , pointing to affected protein features and splice sites. The A259G variant was also

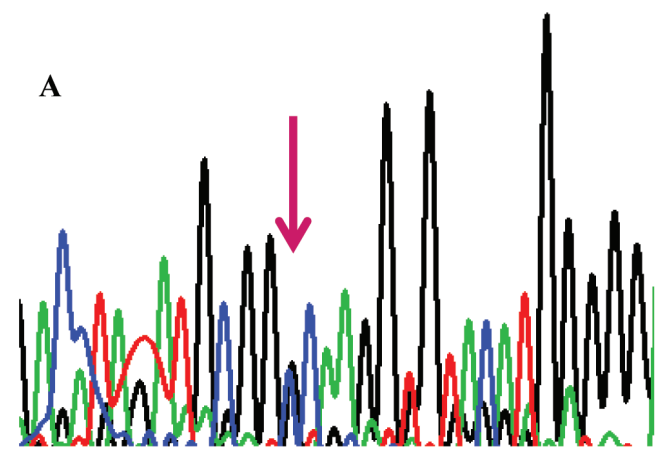

Alanine 259 Glycine

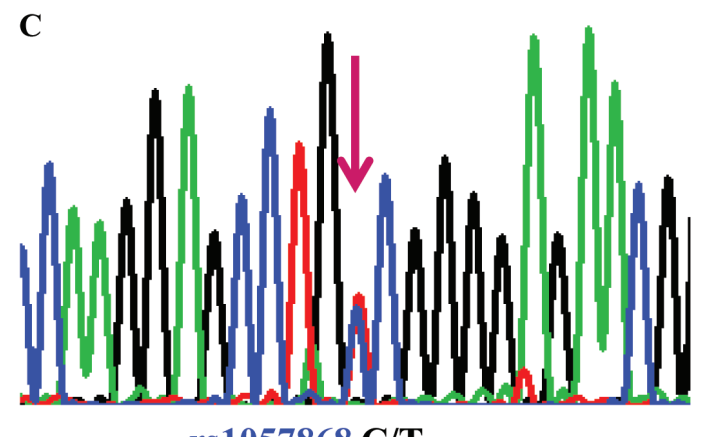

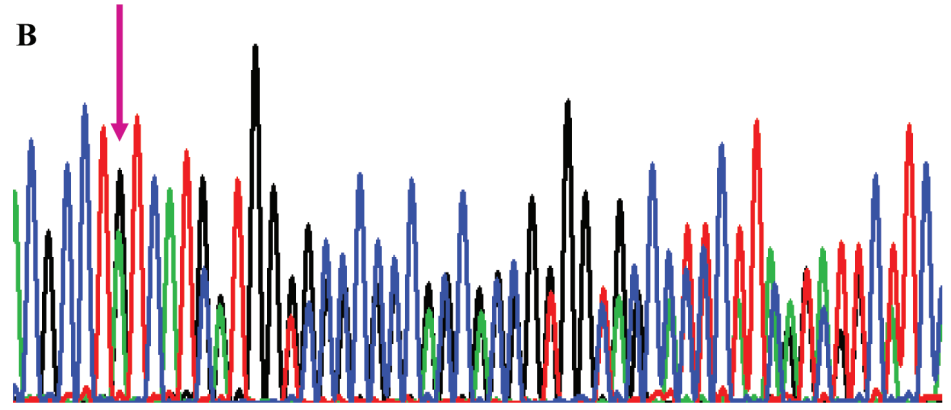

Del 1696_1698delGTC > del531Valine

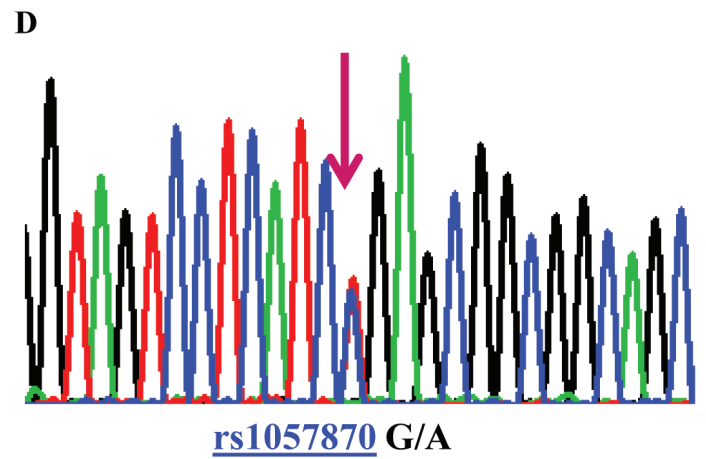

Figure 2. Electrochromatograms showing P450 oxidoreductase's identified genetic alterations (A and B) and the two polymorphisms (C, D). 
named as disease causative by Hansa application, although analysis of this aminoacidic substitution in SIFT defined it as a tolerant change.

No nucleotide changes were detected in the 8 exons of P450c17.

The mutation numbering was carried out by referring to the appropriate National Center for Biotechnology Information (Bethesda, MD) reference sequences [P450 oxidoreductase, NM_000941.2 and NP_000932 (protein).

It was not possible to obtain parental DNA in order to compare our findings and provide information about the inheritance.

\section{DISCUSSION}

It is very unusual to come across a case of ambiguous genitalia while evaluating infertility in an adult male. Despite the fact that the patient in question had previously been evaluated by several doctors, attention had been focused on hormonal and semen determinations without a careful physical examination. The patient's insistence on preserving fertility had apparently impeded an accurate diagnosis. Although it is extremely rare for ambiguous genitalia to be discovered in an adult male seeking fertility, physical examination of the external genitalia should never be omitted.

The diagnosis was based on the fact that plasma increased 17-OH-Pr and decreased androgens both at baseline as well as after ACTH and hCG stimulation. Urinary steroid determination by gas chromatographymass spectrometry was not performed, initially because our hospital lacked the facilities and then due to our patient's departure abroad.

Since the initial description of cytochrome P450 oxidoreductase deficiency, ${ }^{5}$ more than 50 mutations have been reported, ${ }^{12,13}$ suggesting that this disorder might be quite common. ${ }^{4,14,15}$ The fact that a patient with two apparently null mutations has never been described implies that absence of residual POR activity is incompatible with life. ${ }^{8,13,23}$

POR mutations can lead to an apparent combined 17-hydroxylase/21-hydroxylase deficiency (OMIM 201750) and a broad spectrum of clinical characteris- tics, ranging from severe neonatal skeletal malformations with genital ambiguity [known as Antley Bixler syndrome (ABS); OMIM 207410] to phenotypically minor polycystic ovary syndrome-like features. . $, 2,8,10,24-26$ Milder POR mutations might not be accompanied by $\mathrm{ABS}$, and the steroidogenic defect may present as hypogonadism and/or infertility. ${ }^{1,8,14,27}$

Our patient was diagnosed with congenital adrenal hyperplasia with apparent combined P450c17 and P450c21 deficiency due to mutations in the POR gene. The genotyping revealed the del531Valine, the missense mutation A259G as well as two polymorphisms: rs1057868 (A503V) and rs1057870 (S572S). However, the unavailability of parental DNA means that there is, unfortunately, no information as to their allelic distribution.

The deletion del531Valine as well as the A259G change have not been previously reported. In silico analysis defined these aminoacidic alterations as disease-causative, but the confirmative data for their pathogenicity would be provided by in vitro assays that would measure the variants' enzymatic activity while determining the variants' allelic localization. Our lack of the required laboratory facilities, resulting in the non-availability of in vitro data, is a limitation of the study.

Regarding the A503V variant, by far the most common POR polymorphism with an overall incidence of $27.9 \%,{ }^{15,28}$ there is a discrepancy between the reported measurements of its activity depending on the assay used. In the classic, non-physiologic assay based on cytochrome c, the ability of A503V to reduce cytochrome $\mathrm{c}$ and its ability to oxidize NADPH was $67 \%$ and $56 \%$ of WT, respectively. ${ }^{28}$ Measurements of the activity of A503V to support catalysis by steroidogenic enzymes varied: A503V had 68\% of WT ability to support the 17-hydroxylase activity of human P450c17 and 58\% of WT ability to support the 17,20 lyase activity of P450c17. ${ }^{8,28}$ By contrast, A503V had $80 \%$ of WT activity to support the 21 -hydroxylation of progesterone by human P450c21, and $95 \%$ of WT ability to support the 21-hydroxylation of 17-OH-progesterone. ${ }^{29}$ Thus, although A503V is evidently not a modifier gene, since it only moderately impaired the metabolism of testosterone, ${ }^{15,30}$ it could be assigned a synergistic role ${ }^{12}$ in causing disease. 
In conclusion, we feel that the importance of carrying out a clinical examination of particularly this type of patient must be underlined, since it is capable of yielding highly significant initial information which, especially in cases revealing evident defects, can assist or direct the process of diagnosis.

\section{GRANTS OR FELLOWSHIP SUPPORTS}

None

\section{ANY CONFLICT OF INTEREST}

None

\section{REFERENCES}

1. Fluck CE, Tajima T, Pandey AV, et al, 2004 Mutant $\mathrm{P} 450$ oxidoreductase causes disordered steroidogenesis with and without Antley-Bixler syndrome. Nat Genet 36: 228-230.

2. Miller WL, Huang N, Fluck CE, Pandey AV, 2004 P450 oxidoreductase deficiency. Lancet 364: 1663.

3. Pandey AV, Fluck CE, 2013 NADPH P450 oxidoreductase: structure, function, and pathology of diseases. Pharmacol Ther 138: 229-254.

4. Pandey AV, Sproll P, 2014 Pharmacogenomics of human P450 oxidoreductase. Front Pharmacol 5: 103.

5. Peterson RE, Imperato-McGinley J, Gautier T, Shackleton C, 1985 Male pseudohermaphroditism due to multiple defects in steroid-biosynthetic microsomal mixed-function oxidases. A new variant of congenital adrenal hyperplasia. N Engl J Med 313: 1182-1191.

6. Shen AL, O'Leary KA, Kasper CB, 2002 Association of multiple developmental defects and embryonic lethality with loss of microsomal NADPH-cytochrome P450 oxidoreductase. J Biol Chem 277: 6536-6541.

7. Otto DM, Henderson CJ, Carrie D, et al, 2003 Identification of novel roles of the cytochrome $\mathrm{p} 450$ system in early embryogenesis: effects on vasculogenesis and retinoic Acid homeostasis. Mol Cell Biol 23: 6103-6116.

8. Huang N, Pandey AV, Agrawal V, et al, 2005 Diversity and function of mutations in p450 oxidoreductase in patients with Antley-Bixler syndrome and disordered steroidogenesis. Am J Hum Genet 76: 729-749.

9. Dhir V, Ivison HE, Krone N, et al, 2007 Differential inhibition of CYP17A1 and CYP21A2 activities by the P450 oxidoreductase mutant A287P. Mol Endocrinol 21: 1958-1968.

10. Pandey AV, Kempna P, Hofer G, Mullis PE, Fluck CE, 2007 Modulation of human CYP19A1 activity by mutant NADPH P450 oxidoreductase. Mol Endocrinol 21: 2579-2595.

11. Fluck CE, Mallet D, Hofer G, et al, 2011 Deletion of
P399_E401 in NADPH cytochrome P450 oxidoreductase results in partial mixed oxidase deficiency. Biochem Biophys Res Commun 412: 572-577.

12. Fukami M, Nishimura G, Homma K, et al, 2009 Cytochrome $\mathrm{P} 450$ oxidoreductase deficiency: identification and characterization of biallelic mutations and genotypephenotype correlations in 35 Japanese patients. J Clin Endocrinol Metab 94: 1723-1731.

13. Scott RR, Miller WL, 2008 Genetic and clinical features of p450 oxidoreductase deficiency. Horm Res 69: 266-275.

14. Sahakitrungruang T, Huang N, Tee MK, et al, 2009 Clinical, genetic, and enzymatic characterization of P450 oxidoreductase deficiency in four patients. J Clin Endocrinol Metab 94: 4992-5000.

15. Miller WL, Agrawal V, Sandee D, et al, 2011 Consequences of POR mutations and polymorphisms. Mol Cell Endocrinol 336: 174-179.

16. Krone N1, Reisch N, Idkowiak J, et al, 2012 Genotypephenotype analysis in congenital adrenal hyperplasia due to P450 oxidoreductase deficiency. J Clin Endocrinol Metab 97: E257-267.

17. Yang J, Cui B, Sun S, et al, 2006 Phenotype-genotype correlation in eight Chinese 17alpha-hydroxylase/17,20 lyase-deficiency patients with five novel mutations of CYP17A1 gene. J Clin Endocrinol Metab 91: 3619-3625.

18. Arlt W, Walker EA, Draper N, et al, 2004 Congenital adrenal hyperplasia caused by mutant $\mathrm{P} 450$ oxidoreductase and human androgen synthesis: analytical study. Lancet 363: 2128-2135.

19. Schwarz JM, Cooper DN, Schuelke M, Seelow D, 2014 MutationTaster2: mutation prediction for the deepsequencing age. Nat Methods 11: 361-362.

20. Ng PC, Henikoff S, 2003 SIFT: Predicting amino acid changes that affect protein function. Nucleic Acids Res 31: 3812-3814.

21. Acharya V, Nagarajaram HA, 2012 Hansa: an automated method for discriminating disease and neutral human nsSNPs. Hum Mutat 33: 332-337.

22. Acharya V, Nagarajaram HA, 2013 Response to: Statistical analysis of missense mutation classifiers. Hum Mutat 34: 407.

23. Fukami M, Horikawa R, Nagai T, et al, 2005 Cytochrome P450 oxidoreductase gene mutations and Antley-Bixler syndrome with abnormal genitalia and/or impaired steroidogenesis: molecular and clinical studies in 10 patients. J Clin Endocrinol Metab 90: 414-426.

24. Pandey AV, 2006 Biochemical analysis of mutations in P450 oxidoreductase. Biochem Soc Trans 34: 1186-1191.

25. Miller WL, 2004 P450 oxidoreductase deficiency: a new disorder of steroidogenesis with multiple clinical manifestations. Trends Endocrinol Metab 15:311-315.

26. Miller WL, Huang N, Pandey AV, Fluck CE, Agrawal V, 2005 P450 oxidoreductase deficiency: a new disorder of steroidogenesis. Ann N Y Acad Sci 1061: 100-108.

27. Hershkovitz E, Parvari R, Wudy SA, et al, 2008 Ho- 
mozygous mutation G539R in the gene for P450 oxidoreductase in a family previously diagnosed as having 17,20-lyase deficiency. J Clin Endocrinol Metab 93: 3584-3588.

28. Huang N, Agrawal V, Giacomini KM, Miller WL, 2008 Genetics of P450 oxidoreductase: sequence variation in 842 individuals of four ethnicities and activities of 15 missense mutations. Proc Natl Acad Sci U S A 105: 1733-1738.
29. Gomes LG, Huang N, Agrawal V, Mendonca BB, Bachega TA, Miller WL, 2008 The common P450 oxidoreductase variant $\mathrm{A} 503 \mathrm{~V}$ is not a modifier gene for 21-hydroxylase deficiency. J Clin Endocrinol Metab 93: 2913-2916.

30. Miller WL, Huang N, Agrawal V, Giacomini KM, 2009 Genetic variation in human P450 oxidoreductase. Mol Cell Endocrinol 300: 180-184. 